\title{
Cellulose-Based Bioelectronic Devices
}

\author{
Ana Baptista, Isabel Ferreira and João Borges
}

Additional information is available at the end of the chapter

http://dx.doi.org/10.5772/56721

\section{Introduction}

The integration of biomolecules with electronic elements to form multifunctional devices has been recently the subject of intense scientific research. The need of new sensors exhibiting a high selectivity and a total reliability in connection with smart systems and actuators for real time diagnostic and monitoring of diseases has driven wonderful developments in sensors and particularly in biosensors. Biosensors can be regarded as complementary tools to classical analytical methods due to their inherent simplicity, relative low cost, rapid response and proneness to miniaturization, thereby allowing continuous monitoring. They can integrate portable and implantable devices and be used in biological and biomedical systems. However, the development of biocompatible, nontoxic and lightweight power sources devices is still challenging. It would enable the production of various functional devices mechanically flexible and auto-sustained, allowing their integration into a wide range of innovative products such as in implantable medical devices.

\section{Bioelectronics}

Bioelectronics is a new multidisciplinary scientific area that results from the combination of biology, electronics and nanotechnology. Multifunctional devices can be made by integrating biological materials with electronic elements providing a novel and broad platform for biochemical and biotechnological processes. These functional devices can be used to develop sensing devices, such as enzyme-based biosensors [1], DNA-sensors [2], immunosensors [3], and to develop implantable biofuel cells [4] for biomedical applications, self-powered biosensors [1], autonomously operated devices, among others.

\subsection{Biosensors}

Functional devices can successfully convert (bio)chemical information into electronic one by means of an appropriate transducer which contains specific molecular recognition 
structures. In this way, biosensors can be described as integrated receptor-transducer devices which provide selective quantitative or semi-quantitative analytical information using biological recognition elements. The main advantages of biosensors, over traditional analytical detection techniques, are their cost-effectiveness, fast and portable detection, which makes in situ and real time monitoring possible. Implantable biosensors can made a continuous monitoring of metabolites providing an early signal of metabolic balances and assist in the prevention and cure of various disorders, for instance diabetes and obesity [5].

Enzymes are well-known biological sensing materials used in the development of biosensors due to their specificity. However, since they have poor stability in solution, enzymes need to be stabilized by immobilization. Enzyme immobilization can be made by covalent linkage, physical adsorption, cross-linking, encapsulation or entrapment [6, 7]. The choice of the immobilization method depends on the nature of the biological element, the type of transducer used, the physicochemical properties of the analyte and the conditions in which the biosensor should operate [8,9]. Moreover, it is essential that the biological element exhibit maximum activity in its immobilized environment.

As a result, the development of a sensing device based on enzymes is in a good agreement with the present concerns of Green Chemistry due to inherently being a clean process. Notwithstanding some shortcomings such as high sensitivity to environmental factors (like $\mathrm{pH}$, ionic strength and temperature), dependence on some cofactors and limited lifetime hinder the utilization of enzymes in some specific situations.

To overcome the drawbacks, enzyme-free biosensors have been actively developed owing to their simple fabrication, stability and reproducible characteristics. Novel nanoparticle (NP)modified electrodes and other functionalized electrodes have been tested in the design of enzyme-free biosensors [10,11]. Nanostructured materials have the advantage to be easily functionalized exhibiting high electrocatalytic activity and stability. For instance, carbonbased nanostructures have been widely studied as a platform which can hybridize with other functionalized materials, such as metal and metal oxides, forming nanocomposites with improved electrochemical properties [12]. Overall, these nanostructures can provide optimal composite electrode materials for high-performance enzyme-free biosensors.

\subsection{Implantable energy harvesting devices}

The rising interest in Micro Electrical Mechanical Systems (MEMS) due to expanding application areas and new products opportunities, gave rise to the need for reliable and cost effective MEMS, especially in areas such as biosensors, energy harvesting, and drug delivery $[13,14]$.

Biomedical technology usually requires various portable, wearable, easy-to-use, and implantable devices that can interface with biological systems. Currently, implantable medical microsystems are powered by small batteries with limited lifetime. Although, the scientific progress in this area has enabled a decrease in the electrical requirements of the miniaturized devices, the development of a suitable power source remains a major challenge 
for many devices in the bioengineering and medical fields. (MEMS)-based electrical power generation devices can allow the autonomous operation of implantable biosensors by direct power supply or supplement the existing battery-based power systems. Harvesting energy directly from the environment is one of the most effective and promising approaches for powering nanodevices. Mechanical energy surrounds us in our daily life, taking the form of sonic waves, mechanical vibrations and impacts. These vibrations can be converted into electricity via electrostatic, electromagnetic, and piezoelectric microgenerators [15-17]. For instance, harvesting energy from the human body can be possible by converting hydraulic energy from blood flow, heart beats and blood vessels contraction [18]. Another consideration is to use body heat to generate electricity using a thermoelectric generator [19].

More recently, biofuel cells have also been considered for energy harvesting. Implantable fuel cell systems, convert endogenous substances and oxygen into electricity by means of a spatially separated electrochemical reaction. Unlike conventional fuels cells, which rely on expensive rare metal catalyst and/or operate on reformed fossil fuels, biofuel cells rely on the chemical reactions driven by diverse biofuels and biological catalyst. Biofuel cells can be classified according to the biocatalyst. Almost all biochemical processes are catalyzed by enzymes. Systems using specific isolated enzymes at least for a part of their operation are known as enzymatic fuel cells [20], while those utilizing whole organisms containing complete pathways are known as microbial fuel cells [21].

After all, energy harvesting devices and their applications are expanding and becoming more attractive especially with advance in microelectronics and MEMS. MEMS-based generation techniques have many characteristics that make them appealing for biological applications, including the ability to control their physical and chemical characteristics on the micrometer and nanometer scale.

\section{Cellulose}

The demand for products made from renewable and sustainable resources, non-petroleum based, and with low environmental safety risk is persistently increasing. For that reason, renewable materials have been widely explored by consumers, industry, and government. Half of the biomass produced by photosynthetic organisms such as plants, algae, and some bacteria is made up of cellulose, which is the most abundant molecule on the planet. Natural cellulose-based materials, such as wood and cotton, have been used by our society as engineering materials for thousands of years. Cellulose exhibit excellent characteristics, which include hydrophilicity, chirality, biodegradability, capacity for broad chemical modification, and ability to form semicrystalline fiber morphologies, which drawn considerably increased interest and encouraged interdisciplinary research on cellulose-based materials.

\subsection{Cellulose source materials}

Cellulose plays a significant role in the structural support of wood, plants, and composites because of its high mechanical properties. Wood remains the most important raw material 
source of cellulose. The structure of wood is highly complex due to the presence of lignin, a three-dimensional polymer network that binds to carbohydrates (hemicellulose and cellulose) to form a tight and compact structure. The compact structure of wood biomass is particularly challenging because in its native state is impossible to dissolve it in conventional solvents. Traditionally, cellulose is extracted from wood through the Kraft pulping process [22] which involves toxic chemicals and the intensive processing conditions. Recently, research studies focused on a "greener" process which uses Ionic Liquids (ILs) for wood dissolution [23]. A wide variety of plant materials have been studied for the extraction of cellulose including cotton, potato tubers, sugar beet pulp, soybean stock, and banana rachis[24, 25]. Furthermore, cellulose microfibrils can be produced by several species of algae, such as green, gray, red, and yellow-green. Among the algae species, differences in cellulose microfibrils structures can be obtained due to the different biosynthesis process [26]. The cellulose obtained from algal species contains porous or spongy like structure, which is substantially different from the higher plant cellulose. Cellulose microfibrils can also be segregate by bacteria under special culturing conditions. Bacteria can produce a thick gel composed by cellulose microfibrils and water ( $97 \%$ of water content). The major advantage found in bacterial cellulose is the possibility to modify microfibrils structure by changing the culture conditions [27].

\subsection{Cellulose functionalization}

The solubility of cellulose depends on many factors especially on its structure, molecular weight and source. Polysaccharides are well-known to manifest a strong tendency to aggregate or to incomplete solubilization due to the formation of hydrogen bonds. The hydrogen bonding patterns in cellulose are considered as one of the most relevant factors on its physical and chemical properties. The solubility, crystalinity and hydroxyl reactivity can be directed affected by intra- and intermolecular bond formation (Figure 1) [28].

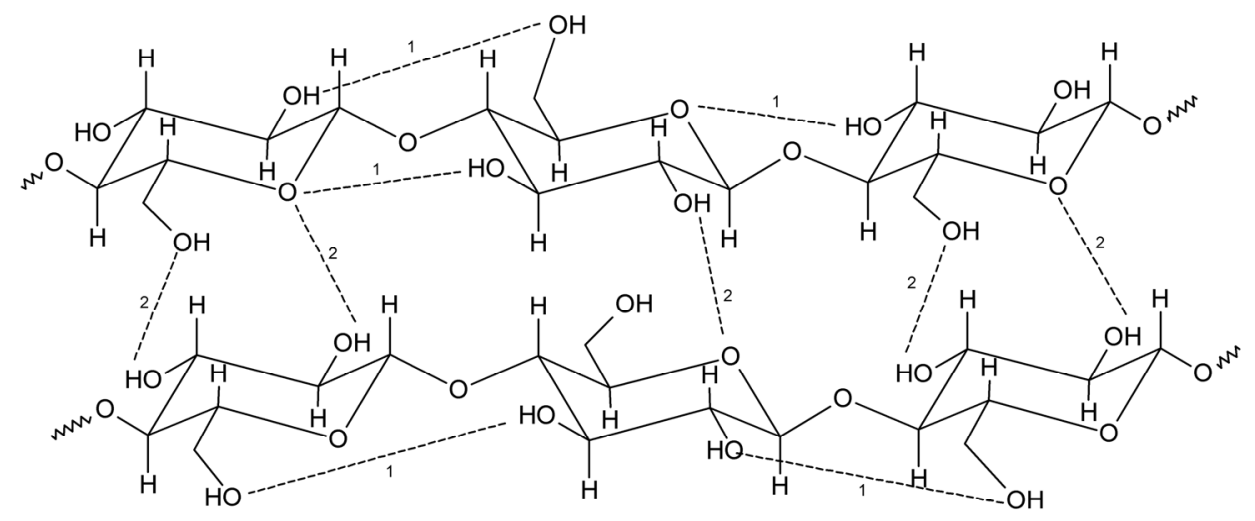

Figure 1. The structure and intra- (1) and interchain (2) hydrogen bonding pattern in cellulose. 
Moreover, cellulose can be chemically modified to yield cellulose derivatives. The cellulose derivatives were designed and fine-tuned to obtain certain desired properties and the chemical functionalization of cellulose is done by changing the inherent hydrogen bond network and by introducing different substituents (Figure 2). Indeed, the properties of cellulose derivatives are mainly determined by the group of substituents and the degree of substitution. These substituents can prevent spontaneous formation of hydrogen bonding or even create new interactions between the cellulose chains. With this insight, recent progress has been made in cellulose chemical modification achieving new routes that are now

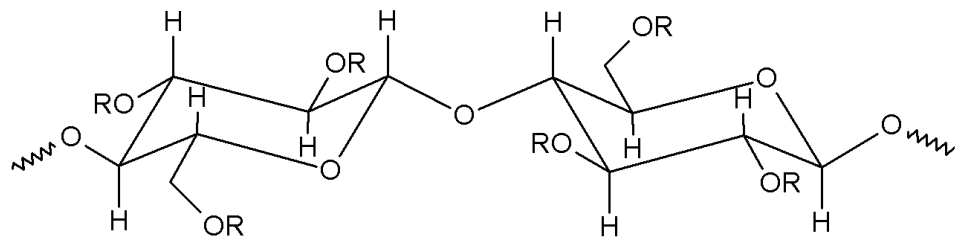

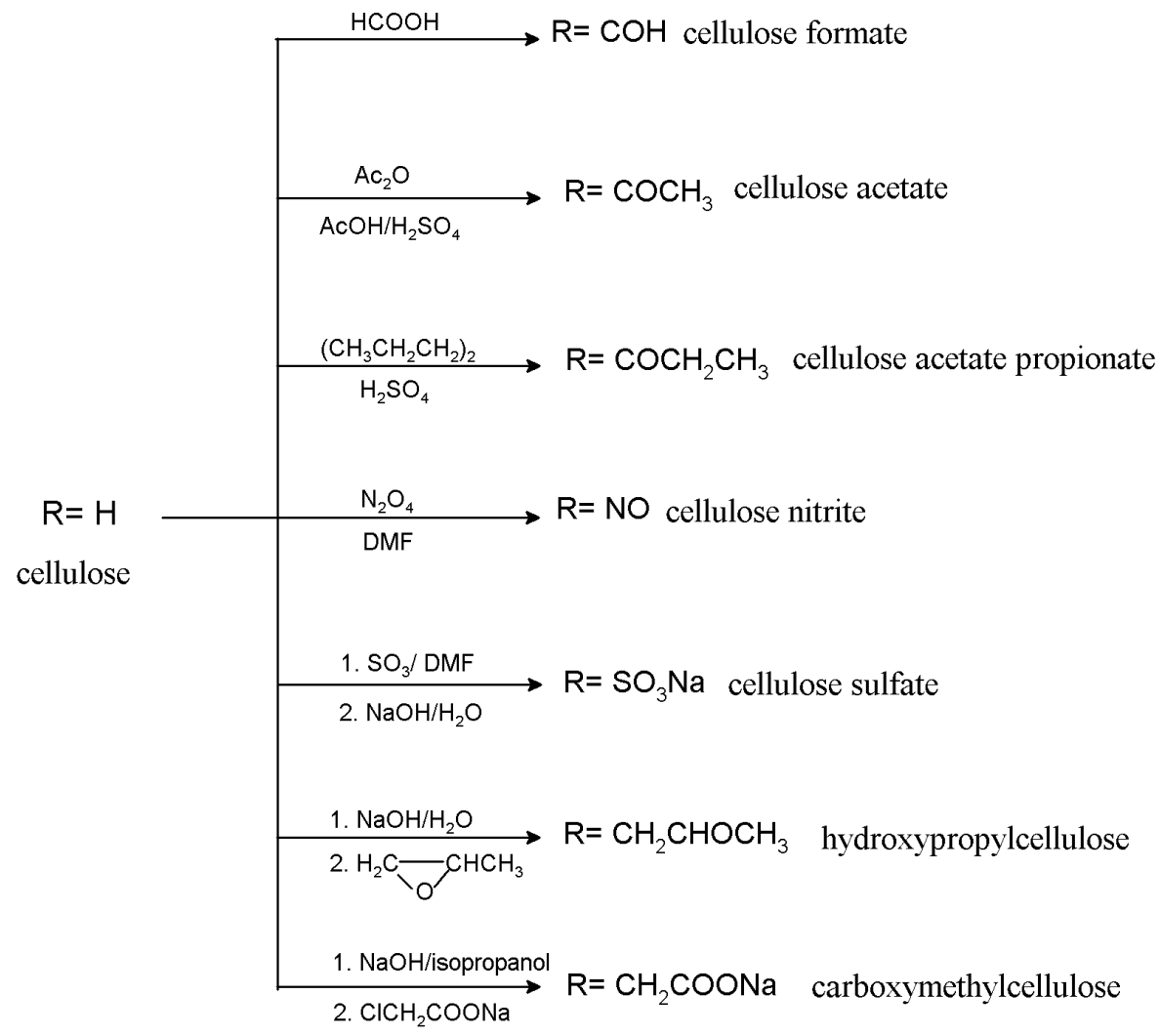

Figure 2. The most relevant cellulose derivatives and their synthesis pathways. 
available for the production of functional and sustainable cellulose-based materials [29]. The chemical modification of cellulose surface is a classical approach to transform the polar hydroxyl groups sitting at the surface of cellulose into moieties able to enhance interactions with the matrix. Indeed, the high density of free hydroxyl groups in cellulose makes it a helpful solid substrate that can undergo functionalization to come into novel advanced applications. Owing to cellulose chain rigidity, some cellulose derivatives can form thermotropic or lyotropic mesophases (in suitable solvents). Among cellulose ethers, hydroxypropylcellulose (HPC) have encouraged the scientific community due to its cholesteric liquid crystalline organization at high concentration [30]. These liquid crystalline phases, with an internal periodic modulation of the refractive index, exhibit many remarkable optical properties as a result of their photonic band structure, which have applications such as polarized light sources, information displays, and storage devices [31]. These phases may also mimic the structural organization of type I collagen and are good analogues of the extracellular matrix, with a structure close to that of biological tissues. These materials can be used either in tissue repair or as models for the culture of cells in 3D, the study of their migration and signaling activities, in a manner close to physiological conditions [32].

In the next section, the functionalization of cellulose will be addressed in detail. Novel functionalized cellulose-based materials have been developed for biosensors and energy storage devices. Some approaches for enzyme immobilization methods including covalent attachment of enzymes by reaction with chemically modified cellulose as well as by adsorption of proteins will be described.

\section{Cellulose-based bioelectronic devices}

\subsection{Cellulose-based matrices for biological immobilization}

Both cellulose and cellulose derivatives, such as cellulose nitrate, cellulose acetate and carboxymethyl cellulose, exhibit an excellent biocompatibility which makes them appropriate for immobilization of biological compounds [33, 34]. As is known, the ideal support for enzymes should be inert, stable and mechanically resistant making the use of cellulose matrices ideal for adsorption and covalent bond immobilization.

The modification of cellulose with dendritic structures is a novel and interesting path to synthesize functional and unconventional cellulose-based supports for the immobilization of enzymes. Moreover, the introduction of reactive groups into the cellulose structure may allow a covalent nonreversible attachment of biomolecules. Maria Montanez [35] and her team suggested the hybridization of cellulose surface with branched dendritic entities that improves the sensitivity toward biomolecules. The described methodology delivers a new toolbox for the design of sophisticated biosensors with advantages such as low detection limit, versatility and suppression of nonspecific interactions providing highly sophisticated cellulose surfaces with unprecedented tunability. Dendrimers are synthetic macromolecules with highly branched structure and globular shape. They possess unique properties such as high density of active groups, good structural homogeneity, internal porosity, and good 
biocompatibility [36]. When addressed to biosensor applications, the well defined dendritic structures generate surfaces with increased reproducibility and high affinity for biomolecular immobilization. This is due to the extraordinary control over the architecture coupled to the possibility of designing a large number of accessible active sites at the periphery of the dendritic scaffolds.

A further approach is the modification of cellulose-based structures with ionic liquids (ILs). Ionic liquids are often used in the preparation of functional materials by its covalent attachment to the support surface forming a stable composite. Moccelini [37] have reported the development of a novel polymeric support based on cellulose acetate and 1-n-butyl-3methylimidazolium bis(trifluoromethylsulfonyl)imide-based IL, BMI.N(Tf)2 IL, for enzyme immobilization. The introduction of the IL probably causes an increase in the distance between the cellulose chains due to the interactions of the anion of the IL and the hydrogen bond networks of the cellulose acetate. Thus, the enzyme can be entrapped within the interstitial space of the formed composite, which results in a considerable stabilization of the enzyme structure, and consequently increases its activity. The study performed demonstrates that this material was able to immobilize Laccase, leading to high efficient and robust biocatalysts thus improving the electrochemical performance of the biosensor.

The use of ILs is an alternative either for cellulose dissolution or to facilitate the dispersion of carbon nanotubes. For that reason, Xuee $\mathrm{Wu}$ [38] describes a method to immobilize enzymes in a cellulose-multiwalled carbon nanotube (MWCNT) matrix via the IL reconstitution process. This method consists in the dissolution of cellulose in the IL, followed by dispersion of MWCNT in the solution and enzyme addition. Subsequently, the IL is removed by dissolution, leaving the cellulose-MWCNT matrix with the enzyme encapsulated on the surface. The cellulose-MWCNT matrix possesses a porous structure which allows the immobilization of a large amount of enzyme close to the electrode surface, where direct electron communication between active site of enzyme and the electrode is enabled. The $-\mathrm{OH}$ groups of cellulose can also provide a good environment for the encapsulation of the enzyme. The authors have employed the resulting porous matrix in the immobilization of Glucose oxidase (GOx). The encapsulated GOx showed good bioelectrochemical activity, enhanced biological affinity as well as good stability.

The simple electrode fabrication methodology and the biocompatibility of the celluloseMWCNT matrix mean that the immobilization matrix can be extended to diverse proteins, thus providing a promising platform for further research and development of biosensors and other bioelectronics devices.

The use of ILs as an intermediary solvent to facilitate the combination of cellulose and CNTs has been suggested by Jun Wan [39]. A cellulose and single wall carbon nanotube (SWNTs) composite was utilized to immobilize leukemia K562 cells on a gold electrode to form a cell impedance sensor.

Envisaging the immobilization of other biomolecules, Alpat and Telefoncu [40] describes the development of a novel biosensor based on the co-immobilization of TBO (Toluidine Blue $\mathrm{O}), \mathrm{NADH}$ (Nicotinamide adenine dinucleotide) and ADH (alcohol dehydrogenase) on a 
cellulose acetate coated glassy carbon electrode for ethanol identification. In fermentation and distillation processes, ethanol can reach toxic concentrations that may cause inflammation and conjunctiva of the nasal mucous membrane and irritation of the skin. Therefore proper detection and quantification of ethanol is of extreme importance. The detector is made by simply deposition on the surface of a glassy carbon electrode and an active layer was prepared by covalent linkage between the mediator TBO and a cellulose acetate membrane. This mediator is commonly used for the oxidation and determination of NADH. Then, a NADH solution and the ADH were added to the cellulose acetate-TBOmodified glassy carbon electrode and tested. The developed biosensor exhibited good thermal stability and long-term storage stability.

The immobilization of proteins on solid surfaces is a key step for the development of medical diagnostic systems. An alternative approach for the immobilization of specific proteins is the chemical modification of cellulose. Stephan Diekmann [41] and his colleagues have described a targeted chemical modification of cellulose to be used as substrate for proteins and biocatalysts bonding. A new cellulose derivative obtained by modification of cellulose with nitrilotriacetic acid (NTA) was used for the complexation of nickel (II). The complex formed was used to immobilize labeled molecules. In that way, the Ni-cellulose derivative allows the development of specific and sensitive molecular diagnostic systems. Another approach is proposed by Jianguo Juang [42] using protein-functionalized cellulose sheets. The surface of the individual cellulose nanofibers was coated with an ultrathin titania gel. The titania coated surfaces were then biotinylated creating a biotin monolayer on each nanofiber by the coordination of carboxyl group. Subsequently, bovine serum albumin (BSA) was added to the functionalized surface to prevent nonspecific adsorption of streptavin. The immobilization of streptavin molecules on its surface was made through biotin-streptavin interaction. Streptavidin has two pairs of binding sites for biotin on opposite's faces of molecule. When immobilized on the cellulose nanofiber with one pair, the other pair is available for further attachment of biotinylated species. The cellulose sheet, composed by numerous nanofibers modified with titania/biotin/BSA layers with anchored streptavidin molecules, gives a large surface area to detect biotin-tagged biomolecules. Thus, biofunctionalized cellulose is a promising substract for specific biomolecular detection.

As previously described, the immobilization of biological compounds can be an important parameter for implantable biosensors due to the fact that it dictates the sensitivity, selectivity and long-term stability of the device. Thus, cellulose appears as an easy functionalized material and an ideal support for adsorption and covalent bond immobilization of biomolecules.

\subsection{Cellulose-based energy storage devices}

There is currently a strong demand for the development of new inexpensive, flexible, lightweight and environmentally friendly energy storage devices. As a result of these needs, research is currently carried out to develop new versatile and flexible electrode materials as alternatives to the materials used in batteries and fuel cells. 
Bacterial cellulose membranes have been widely used as an active layer for the construction of electrodes for fuel cells. Barbara Evans [43] and her colleagues describe the ability of bacterial cellulose to catalyze the precipitation of palladium within its structure. Since bacterial cellulose fibrils are extruded by bacteria and then self-assemble to form a threedimensional network configuration, a structure with a high surface area with catalytic potential is generated. Bacterial cellulose has reducing groups able to promote the precipitation of palladium, and others metals such as gold, and silver from aqueous solution. Then, the metalized bacterial cellulose can be used as anode or cathode in biofuel cells and in biosensors. The possibility of bacterial cellulose to be used for the anodic oxidation of $\mathrm{H}_{2}$ envisaging an energy conversion device has been proved. Another combination of bacterial cellulose and carbon based electrodes was suggested by Yan Liang [44]. He proposes the fabrication of a novel composite based on the combination of carbonized bacterial cellulose nanonofibers and carbon paste electrode. Due to its nanodimension, lower cost and prominent electrochemical properties, bacterial cellulose-based carbonaceous materials would be an ideal candidate for the preparation of novel carbon paste electrodes. A conductive polyaniline (PANI)/bacterial cellulose nanocomposite membrane was reported by Weili $\mathrm{Hu}$ [45]. The author reports on the oxidative polymerization of aniline using the tridimensional structure of the bacterial cellulose as a template. The resulting PANI-coated bacterial cellulose composite formed a uniform and flexible membrane with a high conductivity and good mechanical properties which could be applied in sensors and flexible electrodes.

A different approach is proposed by Xueyan Zhao [46]. He reports on the use of cellulose materials for the preparation of hierarchical carbon materials. A novel method of fabrication of CNT-carbon fibers was developed through carbonization of cellulose fibers being the growth of CNT in the presence of a metal catalyst. A single CNT modified carbon fiber was used as a microelectrode, and then tested for the efficiency of oxidation reaction of NADH (Nicotinamide adenine dinucleotide) generated from the glycerol oxidation reaction. The single fiber microelectrode is promising for applications such as enzyme, glycerol, and NADH biosensors. Also, Sungryul Yun [47] suggests the fabrication of MWCNTs/cellulose composites. In this work, MWCNTs were covalently grafted to cellulose. The covalently grafted MWCNTs improve the mechanical properties of cellulose due to their homogeneous distribution in the composite. Moreover, if MWCNTs can be aligned by the cellulose chains the mechanical properties will be greatly enhanced. Thus, homogeneous distribution of MWCNTs covalently grafted to a cellulose matrix allows the construction of stable electron pathways for cellulose-based electronics and mechanical reinforcing.

Recently, cellulose paper has been (re)discovered as a smart material that can be utilized for sensors and actuators. Celulose-based energy storage devices have significant inherent advantages in comparison with many currently employed batteries and supercapacitors regarding environmental friendliness, flexibility, cost and versatility. The development of cellulose-based flexible energy storage devices is particularly interesting due to the simple procedures for manufacturing these cellulosic composites being, consequently, relatively inexpensive. Various types of devices, such as thin film transistors [48], active matrix 
displays, sensors, batteries [49] and capacitors [50] have been fabricated on paper substrate [51]. Liangbing $\mathrm{Hu}$ [50] and his colleagues have demonstrated that the application of paper can be expanded to energy storage devices by coating it with a simple solution of CNTs. Because paper absorbs solvents easily and binds with CNTs strongly, the fabrication process for the conductive paper is much simpler than that for other substrates, such as glass or plastics. CNTs deposited on porous paper are more accessible to ions in the electrolyte than those on flat substrates which can result in high power density. Because of the high conductivity and the large surface area, the conductive paper was studied in supercapacitors applications as active electrodes and current collectors.

A new design and fabrication method for a supercapacitor based on a flexible CNTcellulose-IL nanocomposite sheets was developed by Victor Pushparaj [52]. They used unmodified plant cellulose dissolved in an IL and subsequently embedded in the MWCNTs. The nanocomposite paper formed, which has a few tens of microns thickness, contains MWCNTs as the working electrode and the cellulose surrounding individual MWNTs, as well as the IL in cellulose, as the self-sustaining electrolyte. In addition to using the IL electrolyte, the authors propose the use of a suite of electrolytes based on body fluids, suggesting the possibility of the device being useful as a dry-body implant. Indeed, the use of biological fluids as an electrolyte for energy applications became an ideal alternative for implantable medical devices and disposable diagnostic kits. The earliest urine-activated paper batteries have been developed and reported by Ki Bang Lee [53]. This device consists in a copper chloride $(\mathrm{CuCl})$-doped filter paper between a copper layer and a magnesium one. Then, the whole assembly is sandwiched between two plastic layers and later laminated by passing it through heated rollers at $120^{\circ} \mathrm{C}$. Magnesium and copper chloride are used as the anode and the cathode of the device respectively, and the $\mathrm{Cu}$ layer acts as an electron-collecting layer. When a droplet of human urine is added to the battery, the urine soaks through the paper between the $\mathrm{Mg}$ and $\mathrm{Cu}$ layers, and after that the chemicals dissolve and react to produce electricity. The chemical composition of urine is widely used as a way of testing various diseases and also as an indicator of a general state of health. For instance, the concentration of glucose in urine can be a useful diagnostic tool for diabetics. Thus, the described work has demonstrated the viability of a urine-activated paper battery for biological application devices including home based health test kits.

Undeniably paper substrates are widely used for flexible electronics not only for being by far the cheapest but also for being one of the most flexible and lightweight material for that purpose. Since paper is manly composed by cellulosic fibers it also exhibits a high surface area which is an advantage for energy applications.

Recently, the electrospinning technique has attracted attention for the preparation of functional materials. Electrospinning is a broadly used technology for electrostatic fiber formation which utilizes electrical forces to produce polymer fibers with diameters ranging from $2 \mathrm{~nm}$ to several micrometers using polymer solutions of both natural and synthetic polymers (Figure 3). This technique allows the production of nanofibers, nanotubes, nanobelts and highly porous membranes. Electrospun nanofibers offer several advantages such as, an extremely high surface-to-volume ratio, tunable porosity, and exhibit a wide 
variety of cross-sectional shapes [31]. Because of these advantages, electrospun nanomaterials have unique properties applicable to a wide range of fields, including the fabrication of nanomaterials for use in energy conversion devices.

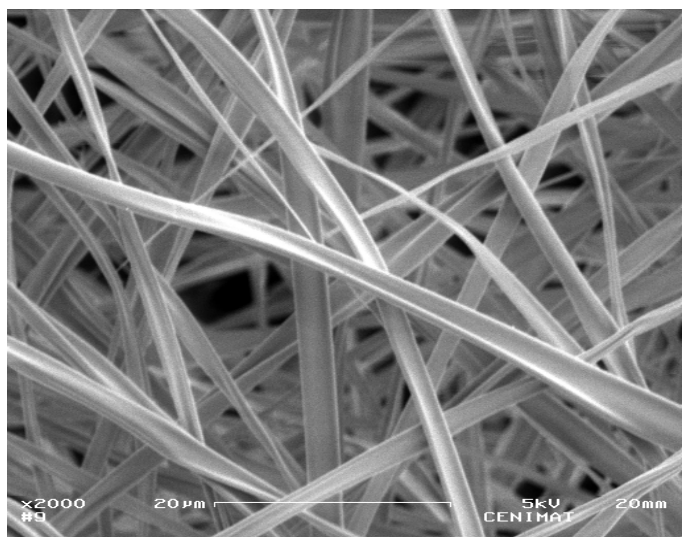

Figure 3. Scanning electron microscopy image of an electrospun cellulose acetate membrane.

Thus, the electrospinning of cellulose and derivatives has been actively studied [31, 54]. Due to their extraordinary properties, such as porosity and large specific surface area, electrospun polysaccharide fibers have been used in biomedical applications such as tissue engineering [55], drug delivery [56], antimicrobial medical implants [57] and biosensors [58, 59].

Liu Shuiping [59] describes the fabrication of photochromic nanofibrous mats through the electrospinning technique. The spiropyrans (SP) are a well-known class of materials that have reversible photochromic properties. On this work a blend solution of cellulose acetate and $\mathrm{NO}_{2} \mathrm{SP}$ (1, 3', 3'-trimethyl-6-nitrospiro (2H-1-benzopyran-2, 2'-indoline) was electrospun forming a homogeneous and highly porous membrane. The photochromic and fluorescent properties of the functionalized nanofibers were determined, showing that the nanofibers exhibited an excellent photosensitivity. These nanofibers have a great potential for application in optical devices and biosensors. Another approach is described by Nafiseh Sharifi [60] selecting the electrospinning technique to develop a nanostructure with electrocatalytic properties. This study focuses on a new, simpler and low cost fabrication method of silver nanostructures by using cellulose as a template. Silver nanoparticles were deposited onto electrospun cellulose fibers followed by thermal removal of the cellulose template. The self-standing silver nanostructure formed is highly porous and exhibited a specific surface area which is in fact appropriate for applications in high surface area electrodes in electrochemistry such as fuel cells.

In fact, the use of electrospun fibers in the development of functionalized materials opens a new path for the creation of novel, lightweight and flexible nanostructures. Our research team is currently working on the development of a bio-battery based on an electrospun cellulose acetate membrane [54]. The bio-battery reported by us is composed by an ultrathin 
monolithic structure in which the separator and the electrodes are physically integrated into a thin and flexible polymeric structure. A highly porous structure is produced by electrospinning to work as a bio-battery after the deposition of metallic layers (electrodes) in each one of the faces (Figure 4). In order to power electronic medical implants, powersupply systems must be able to operate independently over a prolonged period of time, without the need of external recharging or refueling. This cellulose-based structure demonstrated the ability to generate electrical energy from physiological fluids showing a power density of $3 \mu \mathrm{W} . \mathrm{cm}^{-2}$ [54]. This is a really promising achievement since a typical power required for a pacemaker operation is around $1 \mu \mathrm{W}$. Besides the supplying of low power consumption devices, biochemical monitoring systems and artificial human muscles stimulation mechanisms can also be foreseen as potential field of applications where it is desirable this kind of implantable micro power sources.

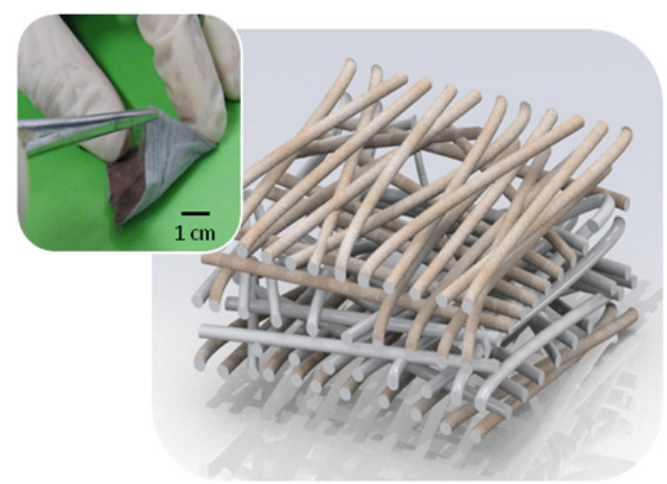

Figure 4. Schematic and macroscopic image of the bio-battery developed by our group. It consists in a cellulose acetate membrane, produced by electrospinning, covered with metallic layers to form the electrodes.

The inspiring advances in the development of innovative cellulose-based bioelectronic devices and its promising perspectives make it a challenging field of study. Electronics can be made lightweight, flexible, and capable of intimate, non-invasive integration with the soft, curvilinear surfaces of biological tissues offering important opportunities for diagnosing and energy harvesting.

\section{Conclusion}

Cellulose and its derivatives have demonstrated to be a versatile material with a unique chemical structure which provides a good platform for the construction of new biomaterials and biodevices. Indeed, the high density of free hydroxyl groups in the cellulose structure makes it a helpful solid substrate that can undergo functionalization allowing the production of new materials for novel advanced applications. From biological immobilization to energy storage devices, the progresses in cellulose functionalization are described as innovative and challenging. Future advances in cellulose-based devices can envisage the development of essential medical implantable devices and healthcare systems. 


\section{Author details}

Ana Baptista, Isabel Ferreira and João Borges*

CENIMAT/I3N and Materials Science Department,

Faculty of Science and Technology of New University of Lisbon (FCT/UNL), Portugal

\section{Acknowledgement}

The authors work was partially supported by Portuguese Science and Technology Foundation (FCT-MCTES) through the Strategic Project PEst-C/CTM/LA0025/2011. Ana Baptista also acknowledges FCT-MCTES for the doctoral grant SFRH/BD/69306/2010.

\section{References}

[1] Katz E, Buckmann A.F, and Willner I (2001) Self-powered enzyme-based biosensors. J. Am. Chem. Soc. 123: 10752-10753

[2] Tersch C, and Lisdat F (2011) Label-free detection of protein-DNA interactions using electrochemical impedance spectroscopy. Electrochimica Acta 56: 7673-7679

[3] Fang X, Tan O.K, Tse M.S, and Ooi E (2010) A label-free immunosensor for diagnosis of dengue infection with simple electrical measurements. Biosensors and Bioelectronics 25: $1137-1142$

[4] Osman M.H, Shah A.A, and Walsh F.C (2011) Recent progress and continuing challenges in bio-fuel cells. Part I: Enzymatic cells. Biosensors and Bioelectronics 26: 3087-3102

[5] Malhotra B.D, and Chaubey A. (2003) Biosensors for clinical diagnostics industry. Sensors and Actuators B. 91: 117-127

[6] Massafera M.P, and Torresi S.I.C (2009) Urea amperometric biosensors based on a multifunctional bipolymeric layer: comparing enzyme immobilization methods. Sensors and Actuators B. 137: 476-482

[7] Zhang B, Weng Y, Xu H, and Mao Z (2012) Enzyme immobilization for biodiesel production. Appl Microbiol Biotechnol. 93: 61-70

[8] Li X, Wang X, Ye G, Xia W, and Wang X (2010) Polystyrene-based diazonium salt as adhesive: A new approach for enzyme immobilization on polymeric supports. Polymer 51: 860-867

[9] Frasconi M, Mazzei F, and Ferri T (2010) Protein immobilization at gold - thiol surfaces and potential for biosensing. Anal Bioanal Chem. 398: 1545-1564

[10] Hua M-Y, Chen H-C, Tsai R-Y, Lin Y-C, and Wang L (2011) A novel biosensing mechanism based on a poly(N-butyl benzimidazole)-modi?ed gold electrode for the detection of hydrogen peroxide. Analytica Chimica Acta 693: 114-120

[11] Lu L-M, Li H-B, Qu F, Zhang X-B, Shen G-L, and Yu R.Q (2011) In situ synthesis of palladium nanoparticle-graphene nanohybrids and their application in nonenzymatic glucose biosensors. Biosensors and Bioelectronics 26: 3500-3504

${ }^{*}$ Correspondig Author 
[12] Yang D-S, Jung D-J, and Choi S-H (2010) One-step functionalization of multi-walled carbon nanotubes by radiation-induced graft polymerization and their application as enzyme-free biosensors. Radiation Physics and Chemistry 79: 434-440

[13] Grayson A.C.R, Shawgo R.S, Johnson A.M, Flynn N.T, Li Y, Cima M.J, and Langer R ( 2004) A BioMEMS Review: MEMS technology for physiologically integrated devices. Proceedings of the IEEE 92 (1): 6-21

[14] Lueke J, and Moussa W.A (2011) MEMS-based power generation techniques for implantable biosensing applications. Sensors 11: 1433-1460

[15] Harb A, (2011) Energy harvesting: State-of-the-art. Renewable Energy 36: 2641-2654

[16] Bouendeu E (2011) A low-cost electromagnetic generator for vibration energy harvesting. IEEE Sensors Journal 11 (1): 107-113

[17] Xu S, Qin Y, Xu C, Wei Y, Yang R and Wang Z.L (2010) Self-powered nanowire devices. Nature Nanotechnology 5: 366-373

[18] Sun C, Shi J, Bayerl D.J, and Wang X (2011) PVDF microbelts for harvesting energy from respiration. Energy Environ. Sci., 4: 4508-4512

[19] Bhatia D, Bairagi S, Goel S, and Jangra M (2010) Pacemakers charging using body energy. J Pharm Bioallied Sci. 2(1): 51-54.

[20] Rincón R.A, Lau C, Luckarift H.R, Garcia K.E, Adkins E, Johnson G.R, and Atanassov P (2011) Enzymatic fuel cells: Integrating flow-through anode and air-breathing cathode into a membrane-less biofuel cell design. Biosensors and Bioelectronics 27: 132-136

[21] Wang H-Y, Bernarda A, Huang C-Y, Lee D-J, and Chang J-S (2011) Micro-sized microbial fuel cell: A mini-review. Bioresource Technology 102: 235-243

[22] Yang L, and Shijie Liu S (2005) Kinetic Model for Kraft Pulping Process. Ind. Eng. Chem. Res. 44: 7078-7085

[23] Wang X, Li H, Cao Y, and Tang Q (2011) Cellulose extraction from wood chip in an ionic liquid 1-allyl-3-methylimidazolium chloride (AmimCl). Bioresource Technology 102: 7959-7965

[24] Dufresne A, Cavaille J-Y, and Vignon M.R (1997) Mechanical behavior of sheets prepared from sugar beet cellulose microfibrils. Journal of Applied Polymer Science 64(6):1185-1194

[25] Zuluaga R, Putaux J-L, Restrepo A, Mondragon I, and Gañán P (2007) Cellulose microfibrils from banana farming residues: isolation and characterization. Cellulose 14: 585-592

[26] Tsekos I (1999) The sites of cellulose synthesis in algae: diversity and evolution of cellulose synthesizing enzyme complexes. J. Phycol. 35: 635-655

[27] Szymańska-Chargot M, Cybulska J, and Zdunek A (2011) Sensing the structural differences in cellulose from apple and bacterial cell wall materials by Raman and FT-IR spectroscopy. Sensors 11: 5543-5560

[28] Kondo T (2005) Hydrogen bonds in cellulose and cellulose derivatives In Severian Dumitriu Editor. Polysaccharides, structural diversity and functional versatility, NY, USA: Marcel Dekker, pp 69-95

[29] Moon R.J, Martini A, Nairn J, Simonsen J, and Youngblood J (2011) Cellulose nanomaterials review: structure, properties and nanocomposites. Chem. Soc. Rev. 40: 3941-3994 
[30] Godinho M.H, Filip D, Costa I, Carvalho A.L, Figueirinhas J.L, and Terentjev E.M (2009) Liquid crystalline cellulose derivative elastomer films under uniaxial strain. Cellulose 16:199-205

[31] Canejo J.P, Borges J.P, Godinho M.H, Brogueira P, Teixeira P.I.C, and Terentjev E.M (2008) Helical Twisting of Electrospun Liquid Crystalline Cellulose Micro- and Nanofibers. Advanced Materials 20(24): 4821-5

[32] Giraud-Guille M.M, Balamie E, Mosser G, Helary C, Gobeaux F, and Vegier S (2008) Liquid crystalline properties of type I collagen: Perspectives in tissue morphogenesis. C. R. Chimie. 11: 245-252.

[33] Frey M.W (2008) Electrospinning Cellulose and Cellulose Derivatives. Polymer Reviews. 48(2): 378-91

[34] Klemm D, Heublein B, Fink H, and Bohn A (2005) Cellulose: Fascinating Biopolymer and Sustainable Raw Material. Angew. Chem. Int. Ed 44(22): 3358-93.

[35] Montanez M.I, Hed Y, Utsel S, Ropponen J, Malmstrom E, Wagberg L, Hult A, and Malkoch M (2011) Bifunctional dendronized cellulose surfaces as biosensors. Biomacromolecules 12(6):2114-25.

[36] Pohl M, Michaelis N, Meister F, and Heinze T (2009) Biofunctional surfaces based on dendronized cellulose. Biomolecules 10: 382-389

[37] Moccelini S.K, Franzoi A.C, Vieira I.C, Dupont J, and Scheeren C.W (2011) A novel support for laccase immobilization: Cellulose acetate modified with ionic liquid and application in biosensor for methyldopa detection. Biosensors and Bioelectronics 26(8):3549-54.

[38] Wu X, Zhao F, Varcoe J.R, Thumser A.E, Avignone-Rossa C, and Slade R.C.T (2009) Direct electron transfer of glucose oxidase immobilized in an ionic liquid reconstituted cellulose-carbon nanotube matrix. Bioelectrochemistry 77(1):64-8.

[39] Wan J, Yan X, Ding J, and Ren R (2010) A simple method for preparing biocompatible composite of cellulose and carbon nanotubes for the cell sensor. Sensors and Actuators B 146: 221-225

[40] Alpat S, and Telefoncu (2010) Development of an alcohol dehydrogenase biosensor for ethanol determination with Toluidine Blue $\mathrm{O}$ covalently attached to a cellulose acetate modified electrode. Sensors 10: 748- 764

[41] Stephan Diekmann S, Siegmund G, Roecker A, and Klemm D.O (2003) Regioselective nitrilotriacetic acid-cellulose-nickel-complexes for immobilisation of His 666 -tag proteins. Cellulose 10: 53-63

[42] Huang J, Ichinose I, and Kunitake T (2006) Biomolecular modification of hierarchical cellulose fibers through tinania nanocoating. Angew. Chem. Int. Ed., 45: 2883-2886

[43] Evans B.R, O'Neill H.M, Malyvanh V.P, Lee I, and Woodward J (2003) Palladiumbacterial cellulose membranes for fuel cells. Biosensors and Bioelectronics 18(7):917-23.

[44] Liang Y, He P, Ma Y, Zhou Y, Pei C, and Li X (2009) A novel bacterial cellulose-based carbon paste electrode and its polyoxometalate-modified properties. Electrochemistry Communications 11: 1018-1021

[45] Hu W, Chen S, Yang Z, Liu L, and Wang H (2011) Flexible electrically conductive nanocomposite membrane based on bacterial cellulose and polyaniline. J. Phys. Chem. 115: $8453-8457$ 
[46] Zhao X, Lu X, Tze W.T.Y and Wang P (2010) A single carbon fiber microelectrode with branching carbon nanotubes for bioelectrochemical processes. Biosensors and Bioelectronics 25: 2343-2350

[47] Sungryul Yun S, and Kim J (2011) Mechanical, electrical, piezoelectric and electro-active behavior of aligned multi-walled carbon nanotube/cellulose composites. Carbon 49: 518-527

[48] Martins R, Barquinha P, Pereira L, Correia N, Gonçalves G, Ferreira I, and Fortunato E (2009) Selective floating gate non-volatile paper memory transístor. Phys. Status Solidi RRL 3 (9): 308- 310

[49] Ferreira I, Brás B, Correia N, Barquinha P, Fortunato E, and Martins R (2010) SelfRechargeable Paper Thin-Film Batteries: Performance and Applications. J. Display Technol. 6: 332-335

[50] Liangbing Hu L, Choi J.W, Yang Y, Jeong S, Mantia F, Cui L-F, and Cui Y (2009) Highly conductive paper for energy-storage devices. PNAS 106(51): 21490-21494

[51] Tobjörk D, and Ronald Österbacka R (2011) Paper Electronics. Adv. Mater. 23: 19351961

[52] Pushparaj V.L, Shaijumon M.M, Kumar A, Murugesan S, Ci L, Robert Vajtai R, Linhardt R.J, Nalamasu O, and Ajayan P.M (2007) Flexible energy storage devices based on nanocomposite paper. PNAS 104 (34): 13574-13577

[53] Lee K.B (2005) Urine-activated paper batteries for biosystems. J. Micromech. Microeng. 15: S210-S214

[54] Baptista A.C, Martins J.I, Fortunato E, Martins R, Borges J.P, and Ferreira I (2011) Thin and flexible bio-batteries made of electrospun cellulose-based membranes. Biosensors and Bioelectronics 26(5): 2742-5

[55] Lee KY, Jeong L, Kang YO, Lee S.J, and Park W.H (2009) Electrospinning of polysaccharides for regenerative medicine. Advanced Drug Delivery Review 61(12):1020-32

[56] Sill T.J, and von Recum H.A (2008) Electrospinning: Applications in drug delivery and tissue engineering. Biomaterials 29(13): 1989-2006

[57] Penchev H, Paneva D, Manolova N, and Rashkov I (2010) Hybrid nanofibrous yarns based on $\mathrm{N}$-carboxyethylchitosan and silver nanoparticles with antibacterial activity prepared by self-bundling electrospinning. Carbohydrate Research 345(16):2374-80

[58] Li J, Vadahanambi S, Kee C-D, and Oh I-K (2011) Electrospun fullerenol-cellulose biocompatible actuators. Biomacromolecules 12(6):2048-54

[59] Shuiping L, Lianjiang T, Weili H, Xiaoqiang L, and Yanmo C (2010) Cellulose acetate nanofibers with photochromic property: Fabrication and characterization. Materials Letters 64 (22): 2427 - 2430

[60] Sharifi N, Tajabadi F, and Taghavinia N (2010) Nanostructured silver fibers: Facile synthesis based on natural cellulose and application to graphite composite electrode for oxygen reduction. International Journal of hydrogen energy 35: 3258-3262 